\title{
IMPACTO DE LAS TIC EN EL TURISMO: CASO COLOMBIANO ${ }^{1}$
}

\author{
Alba Ligia López Rodríguez* \\ Universidad Nacional Abierta y a Distancia (UNAD). Bogotá. Colombia \\ Sergio Andrés López Rodríguez** \\ Universidad Libre Seccional Cali. Colombia
}

\section{RESUMEN}

Las Tecnologías de la Información y la Comunicación (TIC) han impulsado el turismo mundial, con la adopción de modelos empresariales centrados en el Marketing por Internet. Para analizar cómo las MiPyme (Micro, Pequeñas y Medianas empresas) turísticas colombianas utilizan estas tecnologías, se hace un estudio exploratorio y descriptivo acudiendo a fuentes primarias y secundarias, permitiendo a groso modo, concluir que la mayoría no utilizan las TIC adecuadamente, aunque hay un entorno favorable con apoyo del Estado mediante políticas para el desarrollo del turismo y la masificación del Internet.

Palabras clave: Turismo en Colombia; TIC en el Turismo; Viajero Social; Marketing Social y Marketing Experiencial.

\section{Impact of ict in tourism: the colombian case}

\section{ABSTRACT}

The information and communication technologies (ICT) have driven global tourism by adopting business models focused on Internet Marketing. To analyze how Colombian tourist MSMEs (Micro, Small and Medium Enterprises) have used these technologies, we do an

Recibido: 4 de junio de 2016

Devuelto para su revisión: 13 de octubre de 2016

Aceptado: 9 de enero de 2017

* Escuela de Ciencias Básicas, Tecnología e Ingeniería (ECBTI). Universidad Nacional Abierta y a Distancia (UNAD). Carrera 14 No. 14 Sur-23. BOGOTÁ (Colombia) E-mail: alba.lopez@unad.edu.co

** Facultad de Ciencias Económicas Administrativas y Contables. Universidad Libre Seccional Cali. Diagonal 37A, n 3-29. CALI (Colombia).E-mail: sergioa.lopezr@unilibre.edu.co

1 La elaboración de éste artículo fue financiado por la Universidad Antonio Nariño y el proyecto de investigación "Utilización de las Tecnologías de la Información y la Comunicación (TIC) en el sector del turismo", financiado por la Fundación Universidad Autónoma de Colombia, con Acta del Consejo Directivo No. 1708 de septiembre de 2012. Investigadora principal, Alba Ligia López Rodríguez. 
exploratory and descriptive study based on primary and secondary sources, allowing roughly to conclude that most do not use ICT appropriately, although there is government support with policies for tourism development and massive spread of the Internet.

Keywords: Colombian Tourism; ICT in Tourism; Social Traveler; Social Marketing and Experiential Marketing.

\section{INTRODUCCIÓN}

Para empezar, se hace un breve esbozo del sector turismo en Colombia y sus bondades, siguiendo con el impacto que las TIC tienen sobre la innovación en la MiPyme. En cuanto al turismo, es una actividad atractiva por la ventaja comparativa que posee el país, representada en la inmensa riqueza natural y cultural que puesta en valor genera oportunidades para el logro de los objetivos económicos y sociales en regiones apartadas y deprimidas, propiciando el desarrollo integral de dichos territorios. Como lo afirman autores como Cabero (2006); Melgosa (2005); Organización Mundial del Turismo [OMT] (2009), se tiene impacto sobre los lugares visitados y aún más allá, involucrando aspectos económicos, factores de producción de bienes y servicios, aspectos socio-culturales y el entorno natural. Ello ha implicado considerar un enfoque de desarrollo, a través de la política pública, que conlleve a la formulación y aplicación de políticas turísticas desde el nivel local, regional y nacional enmarcados en los acuerdos internacionales que garanticen el Desarrollo Sostenible de los destinos, además de tener estrategias de comunicación para dar a conocer los destinos y transformar así las ventajas comparativas en ventajas competitivas. En Colombia se crea el Programa de Transformación Productiva $\left(\mathrm{PTP}^{2}\right)$, donde se encuentran tres modalidades de turismo (turismo de naturaleza, turismo médico y turismo de bienestar).

Por su parte Gálvez (2014), en su estudio sobre el impacto de las TIC en la innovación de la MyPyme colombiana, asegura que estas nuevas tecnologías son el factor central para alcanzar la competitividad y la sostenibilidad. Pero se requiere la formación de los colaboradores para las actividades de administración, producción, prestación de servicios y gestión tecnológica. Razón por la cual el tema de las TIC se encuentra en las agendas de muchos países, como es el caso de Colombia, donde se crea en 2009 mediante la Ley 1341, el Ministerio de las TIC [MinTIC]) para masificar su uso.

De ahí que el marketing turístico por Internet se convierta en una estrategia relevante para promocionar y lograr destinos de clase mundial, porque éstas herramientas tecnológicas desde la última década del siglo XX han tenido un crecimiento y masificación exponenciales, convirtiéndolas en factores esenciales para el desempeño productivo, el crecimiento económico y el desarrollo social en todos los países, obedeciendo a un cambio de paradigma tecno-productivo (DANE, 2015; Gálvez, 2014; MinTIC, 2014).

2 "El Programa de Transformación Productiva es una de las herramientas dispuestas por el Gobierno Nacional para la ejecución de la Política Industrial que conduzca al Desarrollo Productivo del país y con el que se generan entornos más competitivos y empresas más fuertes y productivas" (Departamento Nacional de Planeación [DNP], 2010:1). 
De manera que la investigación que da origen al presente documento, tiene su origen en dos fenómenos: el crecimiento del turismo y el auge de las TIC. El primero, con una gran capacidad para el logro de los objetivos económicos, sociales y ambientales en los niveles nacional, regional y local; por lo que es apoyado desde la política pública colombiana como sector emergente de alto crecimiento y dotado con estrategias para promover su desarrollo a través del PTP. El segundo aspecto, el auge de las TIC como agente para la consolidación competitiva de las empresas y como estrategia del Estado colombiano que pretende masificar su uso (Gálvez, 2014; MinTIC, 2014). Además, es una realidad que estas nuevas tecnologías han propiciado el surgimiento de formas diferentes de viajar. Según Berné, García, García y Múgica (2011), el viajero social tiene nuevas expectativas que impulsan cambios en las estrategias del marketing hacia lo que se ha llamado "marketing social" y "marketing experiencial", modalidades contempladas dentro del Marketing por Internet.

Para el estudio se acude a la revisión de fuentes secundarias para indagar sobre la influencia de las TIC en la industria del turismo a nivel mundial, especialmente en países, como España, donde el sector está más desarrollado y de ésta manera construir un marco teórico básico. Por otra parte, se indaga sobre las políticas nacionales y sus resultados para la masificación de Internet. También, se acude al estudio de fuentes primarias para caracterizar las TIC que utilizan las empresas colombianas, acudiendo a un instrumento validado que se aplica a una muestra representativa de 99 empresas con una confiabilidad del $90 \%$.

Los resultados, a groso modo, muestran que el turismo es uno de los sectores donde las TIC han cambiado drásticamente la relación entre los turistas y los proveedores de servicios turísticos, migrando hacia el Marketing Social basado en la experiencia (también llamado Marketing Experiencial). También se concluye que se abren oportunidades para que las empresas de turismo en Colombia integren a su estructura empresarial (gestión, marketing y comunicación) las TIC, porque se cuenta con la ayuda del Gobierno Nacional mediante políticas públicas de apoyo al sector a través del Ministerio de las TIC. Según MinTIC (2014), para 2018 la cobertura en los municipios del país con Internet de banda ancha será del $100 \%$. En contraste, el estudio de fuentes primarias dejó ver que la utilización que hacen las empresas de las TIC como estrategias de marketing, es aún bajo para generar los impactos deseados y que las herramientas más utilizadas por las empresas estudiadas son el correo electrónico y la página web (90\% y $70 \%$ respectivamente). Asimismo, aunque el $75 \%$ de ellas, tienen presencia en las redes sociales sólo el $20 \%$ las revisan a diario, pero no hay una estrategia clara al respecto por desconocimiento sobre el tema. Finalmente, se puede afirmar que la presencia de dichas empresas en Internet es relativamente reciente ya que sólo el 29\% de ellas lleva más de 5 años y el $25 \%$ tiene menos de 1 año.

\section{TURISMO Y TIC}

\subsection{Relevancia del turismo}

El turismo es un medio para lograr la transformación social y el desarrollo territorial integral, aspectos deseados para el desarrollo sostenible. Muchos autores coinciden en que éste sector económico, permite disminuir la brecha de inequidad social ya que puede vincular comunidades vulnerables en sitios apartados facilitando la construcción de una nueva 
sociedad, con más arraigo territorial y más cohesión social, contribuyendo a la construcción de una sociedad más justa (PNUMA y OMT, 2002; OMT, 2009; EUROPARC, 2007; Cabero, 2006; Cervantes, Jorge y Gómez, 2007; López y López, 2007; López, 2009; López \& Martínez, 2015; Ramos y Gómez, 2010; Yunis, 2006; entre otros). De ahí que la planificación responsable del destino es necesaria para garantizar que las visitas no excedan la capacidad de carga del destino, donde debe prevalecer la calidad y no la cantidad, esto en concordancia con lo que dicen Meyer (2002); Melgosa (2005) y Perdomo (2007). Solamente los destinos turísticos que se comporten sosteniblemente, serán viables en el futuro y las TIC son un invaluable medio para lograrlo (Vilaseca, Torrent, Lladós y Garay, 2007; Gálvez, 2014).

Por su parte, Moreno (2007) y Geoffrey y Brent (1999), hacen alusión a los efectos positivos que tiene el turismo, donde los beneficios económicos dinamizan en la región receptora una conciencia por valorar e invertir en el desarrollo del destino, promoviendo la recuperación, conservación y puesta en valor del patrimonio turístico como vocación económica del mismo. Además, desde el punto de vista social, hay un incremento en el nivel cultural de la población que se preocupa por el medio ambiente y la conservación de dicho patrimonio.

Pero también hay efectos negativos derivados del turismo mal planificado, donde la afluencia masiva de turistas tiene efectos desastrosos especialmente para aquellos destinos basados en recursos naturales y culturales. Tales efectos pueden ser: a) afectación del patrimonio turístico; b) estímulo a la llegada de nuevos pobladores atraídos por la oportunidad económica, originando la superpoblación del área; c) el menoscabo de la experiencia turística, creando imagen de mala calidad al destino y d) el surgimiento de conflictos sociales, que deterioran la cohesión y el arraigo, empezando de ésta manera un círculo de destrucción que termina con el empobrecimiento de las comunidades y la destrucción del patrimonio natural y cultural (Moreno, 2007; Perdomo, 2007; Yunis, 2006).

Es así como países con alto desarrollo turístico han entendido la importancia de velar por la sostenibilidad y productividad de la actividad, impulsando la investigación, el desarrollo, la tecnología y la innovación para mantener y ampliar el nicho de mercado compuesto por turistas cada vez más exigentes en cuanto a calidad, con mayor dependencia de Internet y con mayor conciencia ambiental (Lamsfus, Grün, Alzua y Werthner, 2010; OMT, 2001; Ramos y Gómez, 2010; Buhalis, 2000).

\subsection{El turismo en Colombia}

Éste sector se ha convertido desde la última década en una actividad cada vez más representativa en la economía nacional, siendo considerado desde la política pública como un sector emergente de alto crecimiento e impacto. De acuerdo a las estadísticas presentadas por el Ministerio de Comercio, Industria y Turismo [MCIT], "el rubro de viajes es el tercer renglón generador de divisas en el país (6.3\%), después de los derivados del petróleo y el carbón (46.9\%)" (MCIT, 2015b: 14). Si se suma el rubro del transporte de pasajeros con una participación del $1.7 \%$, las actividades asociadas al turismo generaron en 2014 el $8 \%$ de las divisas del país. En cuanto a la llegada de turistas, el crecimiento es muy superior al promedio mundial como se aprecia en la Gráfica 1, donde se analiza el período 2004-2014. El promedio del crecimiento anual de las llegadas de turistas en éste lapso de tiempo, en vuelos nacionales fue del 11.2\% e internacionales del 11.7\% (MCIT, 2015a). 


\section{Gráfica 1 \\ LLEGADA DE TURISTAS AL PAÍS EN VUELOS NACIONALES E INTERNACIONALES DURANTE EL PERÍODO 2004-2014}

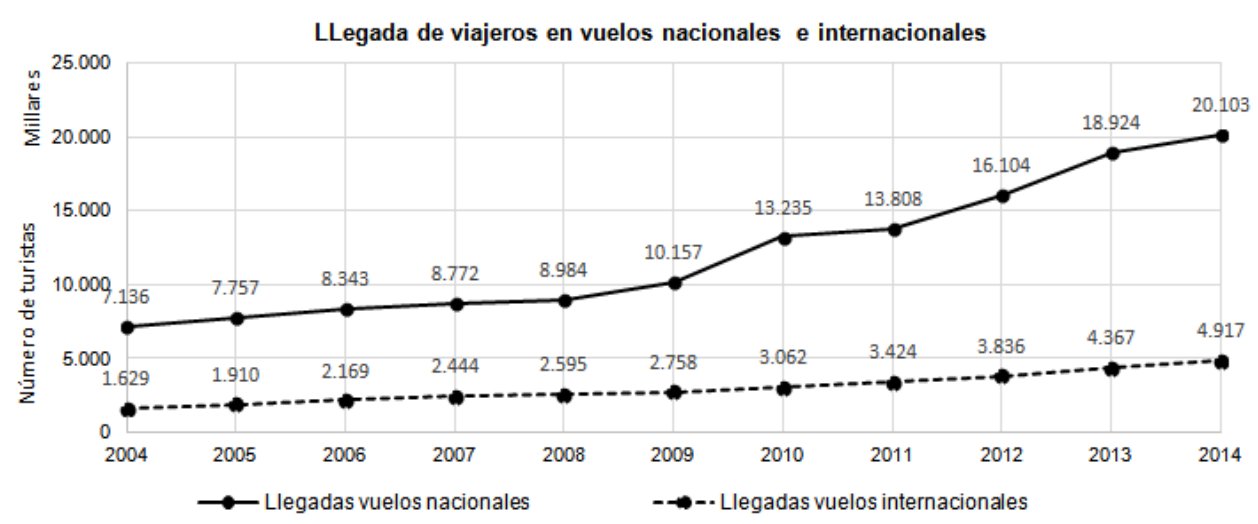

Fuente: elaboración propia a partir de las estadísticas presentadas por MinCIT (2015a).

Para autores como DNP y MCIT (2011), MCIT y Ministerio de Cultura de Colombia [MinC] (2007), DPN (2005), Castrillón (2010) y López y Martínez (2015), entre otros, Colombia posee un enorme potencial turístico por su riqueza natural, biodiversidad y expresiones culturales, que le dan en su conjunto y a muchas regiones en particular una interesante ventaja comparativa en turismo de naturaleza pero que no ha logrado, en la mayoría de las regiones, transformarla en una ventaja competitiva debido a la falta de estrategias de marketing efectivas que den a conocer los productos y destinos turísticos. Esto es relevante porque donde se concentra la mayor parte de la riqueza natural del país son zonas deprimidas, con profundas desigualdades sociales y en la mayoría de los casos genera conflictos en el uso del suelo debido a la explotación inadecuada (minería ilegal; cultivos y ganadería que corren su frontera hacia áreas protegidas y otras prácticas ilegales y depredadoras). Así que esta riqueza natural y cultural podría utilizarse para cambiar la vocación del suelo hacia el turismo sostenible, salvaguardando el invaluable patrimonio natural y cultural, y proveyendo a dichos territorios de herramientas sociales y económicas que les permitan enfrentar el reto del post-conflicto para alcanzar un desarrollo integral y una sociedad más justa y equitativa, como lo plantean la OMT (2001) y Ramos y Gómez (2010).

\subsection{El impacto de las TIC en el turismo}

Internet ha sido la tecnología que mayor impacto ha tenido en las empresas y desde la década de los años 90 se ha convertido más en una necesidad que solamente en un elemento diferenciador. A este respecto autores como Casaló, Flavián y Guinalíu (2012); Akoumianakis et al. (2011); Berné et al. (2011); Lamsfus et al. (2010); Buhalis (2000); Porter (1991); UNCTAD (2005) y Vilaseca et al. (2007), consideran que la irrupción 
hacia una economía basada en el conocimiento (etapa post-fordista ó revolución de la información), fundamentada en la globalización, donde las TIC han cambiado la forma de competir de las empresas, imponen un nuevo paradigma y son la base material de lo que se llama "la tercera revolución industrial". Para el caso del turismo, que en su mayoría son servicios, la innovación es un factor diferenciador del mercado y la adopción de las TIC, ha tenido una alta aceptación en todas las etapas del ciclo turístico. En este sentido, Vilaseca et al. (2007: 219) afirma: "que cuanto más maduro se encuentra un servicio, mayor capacidad de innovación dispone" y para Buhalis (2000) aprovechar las nuevas tecnologías e Internet permite a los destinos mejorar su competitividad mediante el aumento de su visibilidad, reduciendo los costos y mejorando la cooperación local.

El alto crecimiento del turismo a nivel mundial, se ha favorecido, entre otros, por el uso de las TIC y por la aparición de los viajes low cost, que han posibilitado el desplazamiento de personas que no lo podrían hacer por los medios tradicionales (Berné et al., 2011; Díaz y López (2016); Clopatofsky, 2013; Jiménez, 2011; Lamsfus et al., 2010; Ramos, 2008; OMT, 2011 y OMT, 2013). Por su parte, la OMT (2012: 2) afirma que según la tendencia de largo plazo estimada en el estudio Tourism Towards 2030, "el número de llegadas de turistas internacionales en el mundo crecerá en promedio un $3.3 \%$ al año entre 2010 y 2030 (...), lo cual llevaría a alcanzar un total de 1.800 millones de llegadas en 2030".

Pasando al tema empresarial, el turismo es un sector intensivo en información y las TIC han cambiado en la forma de relacionarse con los clientes (turistas o visitantes) provocando cambios profundos en la gestión e infraestructura de las empresas; esto ha generado, por una parte, un notable mejoramiento de la capacidad productiva y competitiva de las que han sabido aprovechar estos avances tecnológicos y por la otra, la desaparición de aquellas que no los han adoptado.

Relacionado con esto, UNCTAD (2005: 1), informa que una gran proporción de las ganancias del turismo, parte de los países en desarrollo y se revierte en grandes multinacionales del sector. No obstante, "Internet brinda una oportunidad para cambiar esta pauta ya que los proveedores turísticos (...) pueden acceder directamente a los clientes". Para Berné et al. (2011: 12), el auge turístico ha propiciado la existencia de diferentes niveles de intermediación, siendo "los distribuidores mayoristas los que canalizan la mayor parte de las contrataciones desde la promoción y la negociación del servicio".

Sin embargo, con la aparición de Internet, hay un cambio en el modelo de negocio de las empresas prestadoras de servicios turísticos porque los proveedores, distribuidores y consumidores pueden tener una comunicación directa mediante las aplicaciones tecnológicas de los dispositivos móviles (teléfonos inteligentes, tabletas y computadores portátiles) surgiendo el nuevo paradigma del marketing: "marketing social y experiencial”, basado en el nuevo perfil de los viajeros, usuarios regulares de Internet que valoran altamente la experiencia del viaje y la facilidad de compartirla en tiempo real con sus amigos de las redes sociales (Casaló et al., 2012; Akoumianakis et al., 2011; Buhalis, 2000 y Lamsfus et al., 2010).

Así, el nuevo paradigma supone el cambio hacia la "Sociedad de la Información" y como consecuencia surgen nuevos hábitos de consumo, convirtiéndose en una variable compleja que modifica drásticamente la estructura del mercado turístico, obligando tanto a las empresas como a los destinos territoriales a adaptarse a las nuevas tecnologías. Algunos de los cambios son: a) aparecen nuevas formas de producción y de prestación de servicios, 
favoreciendo la creación de MiPymes con alta capacidad de flexibilización y personalización de los servicios; b) se genera una oferta diversificada de los destinos turísticos en áreas geográficas antes desconocidas; c) surge un enfoque turístico internacionalizado; d) se estimula el aumento de viajes debido a la oferta de paquetes low cost; e) mayor nivel de personalización en la oferta turística; f) más claridad en la responsabilidad social, la protección al medio ambiente y la solidaridad, y g) aumento del turismo en zonas geográficas apartadas de los litorales, factor favorecido por la expectativa "experiencial" en períodos cortos de ocio -de fin de semana o en los puentes festivos de dos o tres días- (Ramos y Gómez, 2010; Berné et al., 2011 y Lamsfus et al., 2010). De aquí surgen dos temas que vale la pena profundizar: el nuevo perfil del viajero social y el marketing turístico por Internet.

\subsubsection{El perfil del viajero social}

El turista o viajero del siglo XXI se orienta a la búsqueda de experiencias y sensaciones, ya no compra servicios sino "experiencias memorables" que proporcionen valor a través de emociones y sentimientos, a esto se le ha llamado el Dream Society. Las TIC han cambiado los paradigmas, han eliminado fronteras, de manera que una parte de la actividad económica realizada con éstas herramientas tiene lugar entre personas que viven en diferentes países y no necesariamente dentro de las fronteras de uno solo (Alvarado, 2013; Formanchuk, 2010; Cerezo, 2010; Jiménez, 2011; Ramos \& Gómez, 2010 y Lamsfus et al., 2010).

El consumidor promedio en Internet muestra a una persona muy informada (en cuanto a precios y productos), que está conectada todo el tiempo, que se relaciona fácilmente con personas de todo el mundo, capaz de desarrollar sus propios contenidos, más cauta a la hora de gastar y con un considerable control sobre la imagen y la comunicación de las marcas. Así, el consumidor por Internet puede ser un excelente "prescriptor", es decir, aquel consumidor que puede promocionar una marca (o un destino turístico) si ha tenido buenas experiencias con el producto o servicio porque tiene una gran influencia sobre sus amigos en la red. En este escenario la calidad de la marca se vuelve un factor clave para crear imagen favorable e impulsar las ventas (Alvarado, 2013) y como lo manifiesta Jiménez (2011), la necesidad de vivir la experiencia es la mayor motivación del viajero social. Por esta razón, los destinos turísticos y las empresas prestadoras de servicios deben enfocarse en proporcionar al viajero todos los medios para que tenga acceso a Internet y que tenga las mejores experiencias, ya que él es el mejor promotor. De ahí, el papel fundamental de las redes sociales donde es tan fácil y divertido promover esta experiencia y sobre todo contarla a los amigos

En este nuevo orden, las intermediaciones están siendo sustituidas. Ahora los viajeros sociales buscan alternativas como compañías low cost y compras on-line, entre otras alternativas que individualicen su experiencia. Aunque se dice que Internet es un enemigo para empresas como las agencias de viaje, lo cierto es que es gran aliado y en España, después de la crisis del 2008 y 2009, se observó un crecimiento de las agencias de viaje, con un híbrido entre la venta directa y la venta por Internet. Estas han aprovechado los beneficios de la red para eliminar canales de intermediación y en la mayoría de las veces negocian directamente con los proveedores, lo que les permite ofrecer precios competitivos y sobre todo el ahorro de tiempo a los clientes que tendrían que navegar por horas para encontrar 
las alternativas que les satisfagan (Botero, Zuluaga y Camacho, 2010; Díaz, 2011; Jiménez, 2011 y López y Martínez (2015). En el 2025 serán muy pocas las personas que no usen Internet y para asuntos de viajes pueden ser asesoradas por sus familiares más jóvenes.

\subsubsection{El marketing por Internet}

Para desarrollar las actividades de marketing por Internet, las empresas cuentan con varios tipos de herramientas que día a día se modernizan, permitiendo una comunicación más directa con los viajeros, internautas y los compradores (Alcocer, 2011; Alvarado, 2013 y Formanchuk, 2010). También pueden ser usadas por los destinos turísticos para promocionar sus productos y atractivos turísticos. En general, los componentes, como se muestran en la Figura 1, se describen a continuación:

- Email-marketing. Es una herramienta de marketing permisivo; aunque no es spam, son correos electrónicos autorizados por las personas, siendo de gran utilidad porque se puede suministrar información más detallada que por otros medios como redes sociales o publicidad simple por Internet.

- Marketing de buscador. Se utilizan los buscadores como Google o Yahoo, entre otros, que posicionan el producto o servicio en los primeros lugares de la búsqueda.

- Marketing de afiliados. En ésta estrategia las empresas o personas externas hacen la labor de promoción del producto o servicio a cambio de un beneficio como contraprestación. Esto se encuentra reglamentado mediante mecanismos legales en varios países.

- Desarrollo del website. Es la estrategia de marketing más utilizada y tradicional. Permite dar al cliente información amplia sobre el producto o servicio, tener control directo sobre los contenidos e instalar herramientas de e-comerce.

- Anuncios on line. Se pagan por click, es decir, se contabilizan las veces que el anuncio es revisado por los internautas. Los buscadores como Google, los diarios y las revistas, entre otros sitios de consulta habitual, los utilizan como un medio de ingresos económicos. Esta estrategia es muy eficiente para hacer llegar el mensaje a un público numeroso.

- Marketing social. Basado en la utilización de las redes sociales, es el que más ha crecido en los últimos años. "Involucra aspectos como la reputación que construye la empresa o en individuo participando como bloguero o en las redes sociales" (López y Martínez, 2015: 62). Para mejorar el impacto del marketing se acude a: blog, podcast, administrar la publicidad boca a boca, optimizar el uso de los medios sociales y estar pendientes de los adelantos tecnológicos para incluirlos. Esta es una realidad compleja y cada empresa puede decidir cuál de estos elementos desea incorporar en su estrategia de marketing. Sin embargo, son las redes sociales el espacio por excelencia preferido por los viajeros. (Casaló, et al., 2012; Alcocer, 2011; Formanchuk, 2010; López y Martínez, 2015; Jiménez, 2011; Nieto, 2012 y Quirós, 2003). Ahora bien, desde el punto de vista del viajero, las etapas, según Jiménez (2011), son: inspiración, planificación, comparación, reserva, viaje y, finalmente, compartir las experiencias. Estas etapas pueden ser cubiertas a través del marketing social. 
Actualmente hay más de 300 redes sociales. Algunas son de tipo regional y otras de tipo global. Se identifican dos grandes grupos de redes sociales, las de entretenimiento como Facebook, Twitter, Fiendster y Habbo, entre las más conocidas en Latinoamérica y las profesionales como Linkedin que agrupa ejecutivos y profesionales; Twitter que además de ser una red de entretenimiento tiene mucha acogida entre los profesionales por su versatilidad de mensajes cortos y microblogs; Youtube, red con alto crecimiento por lo atractivo de los vídeos y Flickr especializada en imágenes- fotos y diapositivas-. (Formanchuk, 2010; Nieto, 2012 y Quirós, 2003).

Por otra parte, el marketing juega un papel destacado en la promoción para consolidar la imagen del destino turístico. En este sentido López y López (2007) y Alcocer (2011), mencionan al marketing social como una estrategia de gestión local, enfocada en tres aspectos: a) la satisfacción de los turistas, más que aumento de los mismos, b) control y evaluación de las relaciones turista- residente y c) el estímulo al desarrollo de la infraestructura del destino para mejorar la experiencia del turista y el bienestar del residente. Para Jiménez (2011), cualquiera que sea el perfil del viajero (social o no social) son sus experiencias las que ayudan a consolidar la imagen del destino. En este sentido el estudio Forrester Research, sobre la efectividad de los medios de promoción y publicidad, afirma que las recomendaciones de boca en boca, es la estrategia más influyente ya que el $94 \%$ de los consumidores confían en recomendaciones y el $84 \%$ de las compras en las empresas aceptan las recomendaciones (referenciado por López y Martínez, 2015). Es recomendable que el marketing social se encuentre dentro de una estrategia de marketing por Internet como las analizadas en la Figura 1, para aprovechar la versatilidad de los otros medios ya que éste incluye lo que López y Martínez (2015) han definido como 6 C: Comunidades, Conversaciones, Contenido, Continuidad, Contexto y Control.

\section{Figura 1}

\section{COMPONENTES DEL MARKETING POR INTERNET}

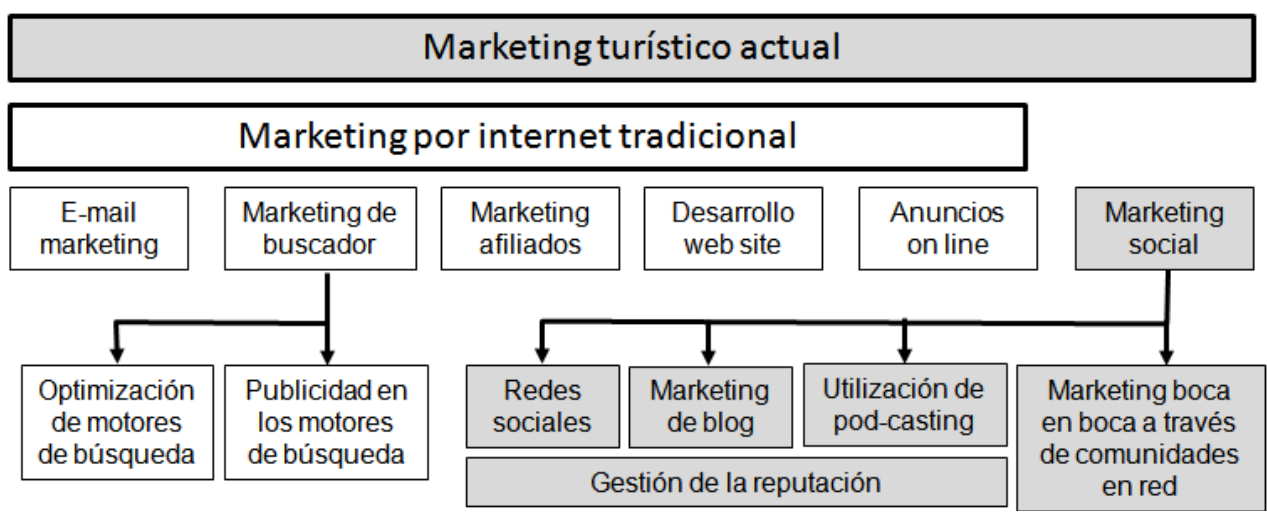

Fuente: elaboración propia a partir de López y Martínez (2015).

En lo que se refiere al caso colombiano, en los siguientes acápites se expone el resultado del estudio. 


\section{DIAGNÓSTICO DE LA UTILIZACIÓN DE LAS TIC EN LA MIPYMES TURÍSTICAS COLOMBIANAS}

Considerando que en el entorno nacional hay apoyo desde lo público y privado para el desarrollo de los sectores fundamentales, el turismo y las TIC, la investigación se aborda mediante un estudio exploratorio y descriptivo basado en la revisión de estudios previos, realizados por el Ministerio de las TIC, para indagar sobre el consumo digital en el país y la investigación de fuentes primarias para identificar el estado de aplicación y apropiación de las TIC por parte de las MiPymes turísticas colombianas.

\subsection{Consumo digital en Colombia}

Es política nacional el fortalecimiento de las TIC, por lo que en 2009 mediante la Ley 1341 se crea el Ministerio de las TIC encargado de diseñar, adoptar y promover las políticas, planes, programas y proyectos para estimular el uso de éstas tecnologías. Desde su creación se han emitido dos documentos de política denominada "Plan Vive Digital", para los cuatrienios 2010-2014 y 2014-218; el primero fue reconocido como la mejor política del mundo en el año 2012, ya que Colombia fue el primer país de la región en alcanzar la cobertura de Internet del 100\%, según MinTIC (2014).

Por otra parte, el Ministerio de TIC y la empresa encuestadora Ipsos Napoleón Franco hicieron un estudio de consumo digital en el país en las 13 ciudades más grandes; entre los principales resultados se tienen: el $80 \%$ de las personas utilizan Internet, donde el mayor crecimiento de consumo con respecto a 2010 se dio en los estratos 1 y 2 con el $17 \%$. Otro dato interesante es que el $84.1 \%$ de los usuarios usan el correo electrónico, el $73.3 \%$ las redes sociales y el $67.9 \%$ buscan información general. El $90 \%$ de los visitantes de redes sociales tienen entre 15 y 24 años y el $80 \%$ pertenecen a los estratos 4 y 5 . Además, las personas que más compran por Internet son las de los estratos 4 y 5 con edades entre los 25 y 44 años. En el último trimestre de 2016, la conexión a banda ancha a Internet presentó un incremento del $23.1 \%$; la de telefonía móvil $3 \mathrm{G}$ y $4 \mathrm{G}$, un incremento del $35.6 \%$ y el índice de penetración de telefonía celular creció $20.34 \%$ con respecto al mismo período de 2015. Estos resultados evidencian la gran oportunidad para que las empresas turísticas incrementen su presencia en Internet ((MinTIC e Ipsos, 2013; MinTIC, 2017).

Lo anterior muestra que en el país hay un entorno muy favorable para que las empresas turísticas, especialmente la MiPymes, utilicen las TIC como un nuevo modelo de negocio y que los destinos turísticos se apropien de éstas tecnologías para convertir las ventajas comparativas en ventajas competitivas. Las MiPyme representan un alto porcentaje del tejido empresarial colombiano y en el sector turístico tienen un papel fundamental para lograr el reparto equitativo de los recursos dada su flexibilidad, como lo plantean Triana, Morales, Guarín. y Saldarriaga (2012).

\subsection{Caracterización de las TIC que utilizan las MiPymes turísticas en Colombia}

Teniendo en cuenta las recomendaciones de Hernández, Fernández y Baptista (2010) y Torres y Lastra (2008), se diseña una encuesta de 10 preguntas, como se muestra en la 
Tabla 1, se determina su validez y confiabilidad. De manera que la validez de contenido se obtiene con la revisión bibliográfica identificando las variables para el logro de los objetivos del estudio, derivando en las preguntas de la encuesta; la validez de criterio se comprueba en la evaluación de expertos y la prueba piloto y, finalmente, la validez del constructo se garantiza porque las variables se han utilizado en otras investigaciones como el caso del estudio realizado por Ortega (2011). La confiabilidad del instrumento se hace mediante el coeficiente $\alpha$ de Cronbach. Según lo planteado por los autores mencionados anteriormente, éste coeficiente es un indicador de correlación con valores entre 0 y 1 , donde un valor superior a 0,8 es aceptable, mayor de 0,96 es sumamente confiable y en 1 la confiabilidad es perfecta. Si los valores están por debajo de 0,8 se presenta variabilidad heterogénea en sus medidas, por lo que llevaría a conclusiones equivocadas. Así, en la prueba piloto con expertos, el índice $\alpha$ de Cronbach para las preguntas con una escala de medición ordinal (preguntas 3, 8 y 9) arroja los siguientes valores respectivamente: $0.832 ; 0.811$ y 0.982 . En consecuencia, por presentar un índice superior a 0,8 , los datos son fiables.

Tabla 1

ESTRUCTURA DE LAS PREGUNTAS DE LA ENCUESTA

\begin{tabular}{|c|c|c|}
\hline Variable & Indicadores & Ítems o preguntas \\
\hline \multirow{3}{*}{ 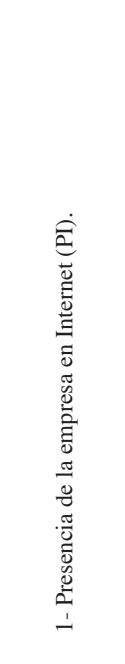 } & Tiempo de PI. & $\begin{array}{l}\text { 1- ¿Desde cuándo la empresa tiene presencia en Internet? } \\
\text { - Menos de un año. } \\
\text { - De } 1 \text { a } 2 \text { años. } \\
\text { - De } 2 \text { a } 3 \text { años. } \\
\text { - De } 3 \text { a } 4 \text { años. } \\
\text { - De } 4 \text { a } 5 \text { años. } \\
\text { - Más de } 5 \text { años. }\end{array}$ \\
\hline & $\begin{array}{l}\text { Visión económica } \\
\text { de la PI. }\end{array}$ & $\begin{array}{l}\text { 2- ¿Cómo entiende la presencia de su organización en Internet? } \\
\text { - Como gasto necesario. } \\
\text { - Como inversión rentable. } \\
\text { - Como inversión con dudas en la efectividad del retorno. } \\
\text { - Como una opción en desarrollo. } \\
\text { - Otro. }\end{array}$ \\
\hline & $\begin{array}{l}\text { Grado en que } \\
\text { considera importante } \\
\text { la PI. }\end{array}$ & $\begin{array}{l}\text { 3- Califique el grado de importancia, para su empresa, de los siguientes aspectos (siendo } \\
1 \text { la menor y } 5 \text { la máxima). } \\
\text { - } \text { Incrementar ventas. } \\
\text { - } \text { Construir marca. } \\
\text { - } \text { Fidelizar clientes. } \\
\text { - Promoción. } \\
\text { - } \text { Comercialización. } \\
\text { - Crecimiento de la empresa. } \\
\text { - Prestigio y reconocimiento social. } \\
\text { - Otro ¿Cuál? }\end{array}$ \\
\hline 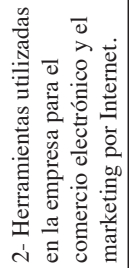 & $\begin{array}{l}\text { Herramientas } \\
\text { digitales utilizadas en } \\
\text { la comercialización } \\
\text { y marketing por } \\
\text { Internet. }\end{array}$ & $\begin{array}{l}\text { 4- De las siguientes herramientas digitales, identifique cuál(es) utiliza su organización. } \\
\text { - Página web. } \\
\text { - Redes sociales. } \\
\text { - Blogs. } \\
\text { - Apps y otras herramientas como redes especializadas en turismo. } \\
\text { - Vídeos, fotos, diapositivas (Youtube, Flickr, otros.) } \\
\text { - Foros. } \\
\text { - OTA (Online Travel Agencies). } \\
\text { - Correo electrónico. } \\
\text { - Otro ¿Cuál?. }\end{array}$ \\
\hline
\end{tabular}




\begin{tabular}{|c|c|c|}
\hline \multirow{3}{*}{ 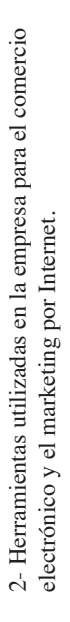 } & $\begin{array}{l}\text { Herramientas } \\
\text { digitales utilizadas en } \\
\text { la comercialización } \\
\text { y marketing por } \\
\text { Internet. }\end{array}$ & $\begin{array}{l}\text { 5- ¿En qué redes sociales tiene presencia su empresa? } \\
\text { - Ninguna. } \\
\text { - Facebook. } \\
\text { - Twitter. } \\
\text { - LinkedIn. } \\
\text { - Youtube. } \\
\text { - Google+ } \\
\text { - Flickr. } \\
\text { - Otra ¿Cuál? }\end{array}$ \\
\hline & $\begin{array}{l}\text { Frecuencia de } \\
\text { actualización de } \\
\text { las herramientas } \\
\text { utilizadas. }\end{array}$ & $\begin{array}{l}\text { 6- ¿Con qué frecuencia actualiza cada una de las herramientas que usa? } \\
\text { Matriz de doble entrada con las mismas herramientas de la pregunta } 4 \text { en el eje Y, y las } \\
\text { opciones del eje X como sigue: } \\
\text { - Diariamente. } \\
\text { - Varias veces a la semana. } \\
\text { - Varias veces al mes. } \\
\text { - Varias veces al año. }\end{array}$ \\
\hline & $\begin{array}{l}\text { Mantenimiento de } \\
\text { las herramientas } \\
\text { empleadas. }\end{array}$ & $\begin{array}{l}\text { 7- ¿Quién se encarga de la actualización de la información en las herramientas de la } \\
\text { anterior pregunta? } \\
\text { - Personal interno. } \\
\text { - Personal externo. }\end{array}$ \\
\hline \multirow{3}{*}{ 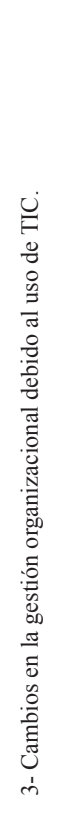 } & $\begin{array}{l}\text { Grado en que } \\
\text { influyen las } \\
\text { herramientas de } \\
\text { marketing online en } \\
\text { las ventas. }\end{array}$ & $\begin{array}{l}\text { 8- Según su percepción, de cada una de las herramientas que utiliza, ¿podría valorar de } \\
1 \text { a } 5 \text { en qué grado influye cada una en las ventas de su empresa? (1= valor más bajo } \\
\text { y } 5=\text { valor más alto). } \\
\text { - Página web. } \\
\text { - Redes sociales. } \\
\text { - Blogs. } \\
\text { - Apps y otras herramientas como redes especializadas en turismo. } \\
\text { - Vídeos, fotos, diapositivas (Youtube, Flickr, otros.) } \\
\text { - Foros. } \\
\text { - OTA (Online Travel Agencies). } \\
\text { - Correo electrónico. } \\
\text { - Otras. Las consideradas en la pregunta } 4 .\end{array}$ \\
\hline & \multirow[t]{2}{*}{$\begin{array}{l}\text { Influencia del uso de } \\
\text { las TIC en la empresa }\end{array}$} & $\begin{array}{l}\text { 9- Para los siguientes aspectos, ¿en qué grado (de } 1 \text { a } 5 \text { ) considera que ha influido el uso } \\
\text { de las TIC en su empresa? ( } 1=\text { valor más bajo y } 5=\text { valor más alto). } \\
\text { - Mayor eficiencia. } \\
\text { - Mayor productividad. } \\
\text { - Mayor competitividad. } \\
\text { - Llegar al público objetivo más fácilmente. } \\
\text { - Diversificación de la oferta. } \\
\text { - Mayor conocimiento de la empresa. } \\
\text { - Otro aspecto ¿Cuál? }\end{array}$ \\
\hline & & $\begin{array}{l}\text { 10- Considera que los cambios en la infraestructura de su empresa para tener presencia } \\
\text { en Internet han sido: } \\
\text { - Ninguno } \\
\text { - Muy bajos } \\
\text { - Bajos } \\
\text { Altos }\end{array}$ \\
\hline
\end{tabular}

Fuente: elaboración propia.

\subsubsection{Población y muestra}

La población se toma del Registro Nacional de Turismo (RNT), el cual es creado mediante la Ley General de Turismo (Ley 300 de 2006) para que las empresas prestadoras de servicios turísticos se inscriban obligatoriamente. De manera que ésta es la fuente más confiable como población de interés para este estudio. El tamaño de la población en 
el momento de hacer el estudio (agosto y septiembre de 2013) está compuesto por las MiPymes con registro vigente en el RNT, y corresponde a 13.727 establecimientos (RNT, 2013 y López y Martínez, 2015). De ellas, el 52.1\% son establecimientos de alojamiento y hospedaje, $29.2 \%$, agencias de viaje, $5.7 \%$ establecimientos de gastronomía y similares, $5.2 \%$ guías de turismo, $1.7 \%$ empresas de transporte terrestre y $7.8 \%$ está compuesto por otros prestadores (empresas de tiempo compartido y multipropiedad; concesionarios de servicios turísticos en parques; arrendadores de vehículos para turismo nacional e internacional; oficinas de representación turística, operadores profesionales de congresos, ferias y convenciones; empresas captadoras de ahorros para viajes y de servicios turísticos; guías de turismo y parques temáticos).

De acuerdo a la composición de la población se toma una muestra representativa con muestreo estratificado, ver más detalles de la población en la Figura 2.

\section{Figura 2 \\ COMPOSICIÓN DE LA POBLACIÓN}

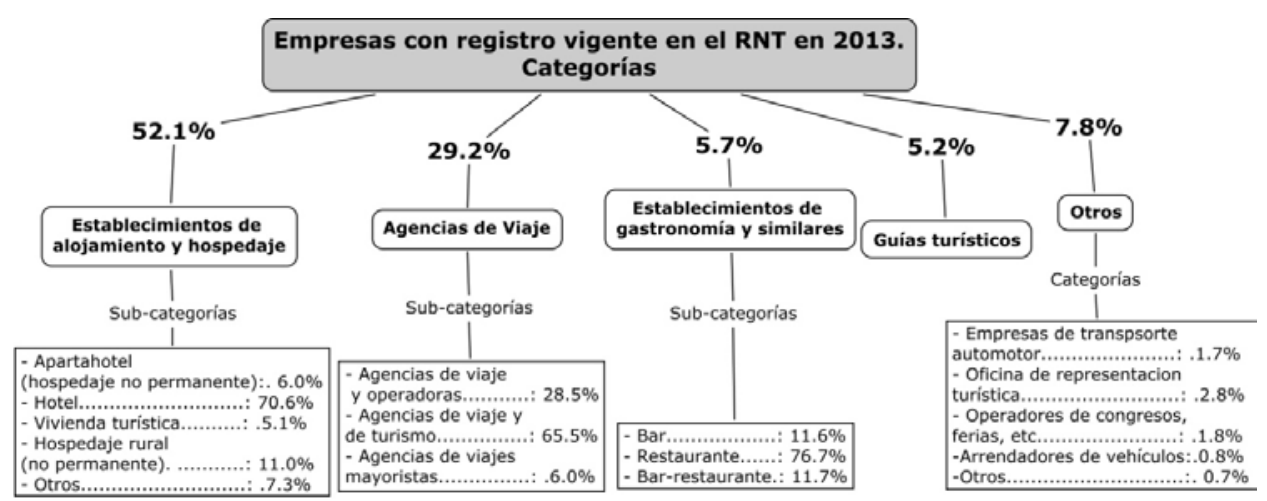

Fuente: elaboración propia a partir de RNT (2013).

El tamaño de la muestra se calcula, según lo recomendado por Hernández, et al. (2010), como sigue:

$\mathrm{N}=$ población $=13727$ establecimientos vigentes en el RNT en 2013.

$\mathrm{z}=$ factor probabilístico $=1.645$ que equivale al nivel de confianza del $90 \%$.

$\mathrm{p}=0.9 ; \mathrm{q}=(1-\mathrm{p}) \mathrm{y} \mathrm{e}=$ error máximo que se permite $=5 \%=0.05$.

Tamaño de la muestra ajustada:

$$
\begin{aligned}
\mathrm{n} & =\frac{\mathrm{n}^{\prime}}{1+\left(\frac{\mathrm{n}^{\prime}-1}{\mathrm{~N}}\right)} \\
\mathrm{n}^{\prime} & =\frac{1.645^{2}(0.9 * 0.1)}{(0.05)^{2}}=97,4 \approx 97 \text { y } \mathrm{n}=\frac{97}{1+\left(\frac{97-1}{13727}\right)}=96,7 \approx 97
\end{aligned}
$$




\subsubsection{Resultados de la aplicación de la encuesta}

Dadas las características del estudio, se utiliza una encuesta online para elaborar y gestionar cuestionarios y se aplica por medio del correo electrónico donde el encuestado accede fácilmente al cuestionario y lo responde vía online. Los hallazgos se resumen a continuación:

En cuanto a la presencia en Internet: la mayoría de las empresas (41\%) llevan entre 2 y 4 años, el 29\% tienen más de 5 años y el 25\% tienen menos de 1 año, lo que evidencia que sus actividades en Internet son relativamente recientes. Además, el $63 \%$ de los empresarios considera que su presencia en Internet es una inversión rentable y el $41 \%$ la consideran como opción en desarrollo.

Sobre las herramientas TIC que más usadas, el 90\% de las empresas acuden al correo electrónico; $75 \%$ redes sociales; $70 \%$ tienen página web; $40 \%$ tienen contenidos de fotos y diapositivas en Youtube, Flickr, entre otras redes; 24\% utiliza apps y otras herramientas como redes especializadas en turismo; $23 \%$ tiene blog; 15\% utiliza OTA (Online Travel Agencies), dirigida a agencias de viaje (29.2\% de las empresas de la muestra), es decir, que solamente la mitad de las agencias de viajes utilizan esta herramienta y otras similares.

Por otra parte, al indagar si las empresas usan estas herramientas para mantener contacto permanente con el cliente, se resalta que el correo electrónico y las redes sociales son revisados diariamente (por el $50 \%$ y $20 \%$ de las empresas respectivamente), mientras que la página web sólo es actualizada varias veces al mes por el $25 \%$ de las empresas. Aunque un grupo apreciable de empresas han empezado a utilizar las herramientas para el marketing, la mayoría no las incorporan como la principal estrategia, sino como un mecanismo más de comunicación. Enfatizando en las redes sociales, las más utilizadas son: Facebook 78\%, Twitter 44\%, Google+ 28\%, Youtube 24\%, LinkedIn 17\%, Flickr 7\%; no utiliza las redes sociales $17 \%$ y otras $8 \%$.

Los resultados sobre la influencia de las TIC en diferentes aspectos organizacionales, mostró valores positivos cercanos a 4/5; el $60 \%$ de las empresas incluyeron otro aspecto adicional que fue el de "Reconocimiento por parte de los clientes". Los resultados se pueden apreciar en la Gráfica 2. Uno de los aspectos relevantes es la influencia sobre las ventas, a este respecto, los empresarios consideran que la página web es la herramienta que más influye, seguida por el correo electrónico (calificación 3.95 y 3.91 respectivamente en la escala de 1-5). En tercer lugar están las apps, calificadas con 3.27 y las redes sociales con 3.0. Más detalle se aprecia en la Gráfica 3. Además, los resultados mostrados en la Gráfica 4 resaltan que los empresarios consideran que las ventas son el aspecto más importante que se beneficia con el uso de TIC, con la mayor calificación (4.64/5.0), seguido por el crecimiento empresarial con 4.48/5.0 y el prestigio de la empresa con 4.42/5.0.

Para concluir, ante la pregunta “¿Cómo considera los cambios en la infraestructura de información de su empresa para tener presencia en Internet?”, el 44\% de los empresarios los consideran altos, el $8 \%$ muy altos, el $25 \%$ bajos y el $11 \%$ muy bajos. Y respecto a la contratación del personal dedicado al mantenimiento de las herramientas utilizadas, el $81 \%$ de las empresas tienen personal interno y el $17 \%$ prefieren contratar a dicho personal. Esto corrobora que para la mayoría de ellas, no hay una estrategia de marketing clara y se acude a los conceptos del marketing tradicional. 


\section{Gráfica 2}

\section{CALIFICACIÓN DE LA INFLUENCIA DE CADA UNA DE LAS HERRAMIENTAS} DIGITALES, EN LA GESTIÓN DE LA EMPRESA. PREGUNTA 9

Resultados promedio de calificación.

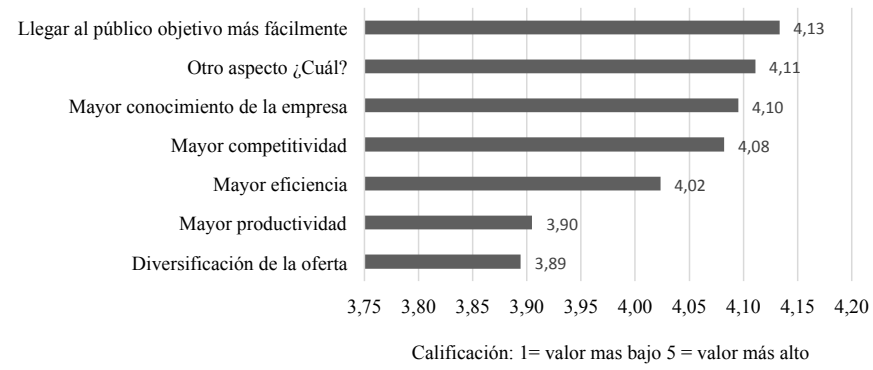

Fuente: elaboración propia.

\section{Gráfica 3}

\section{CALIFICACIÓN DE LA INFLUENCIA DE CADA UNA DE LAS HERRAMIENTAS DIGITALES, EN LAS VENTAS DE LA EMPRESA. PREGUNTA 8}

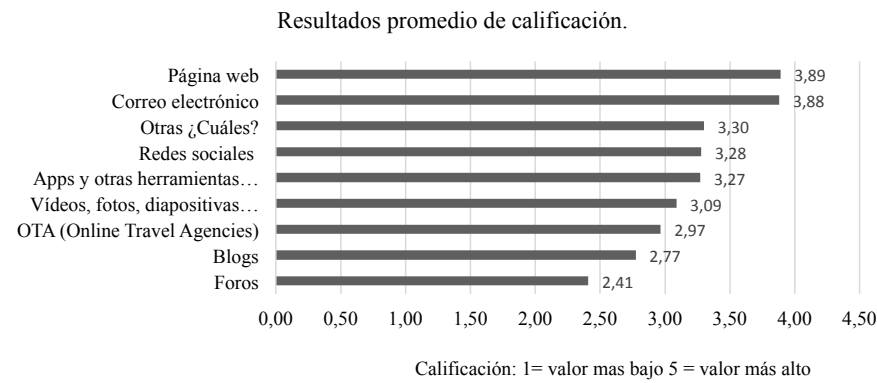

Fuente: elaboración propia.

\section{Gráfica 4 \\ CALIFICACIÓN DE LOS ASPECTOS DEL ÁREA DE MARKETING Y VENTAS. PREGUNTA 3 \\ Resultados promedio de calificación.}

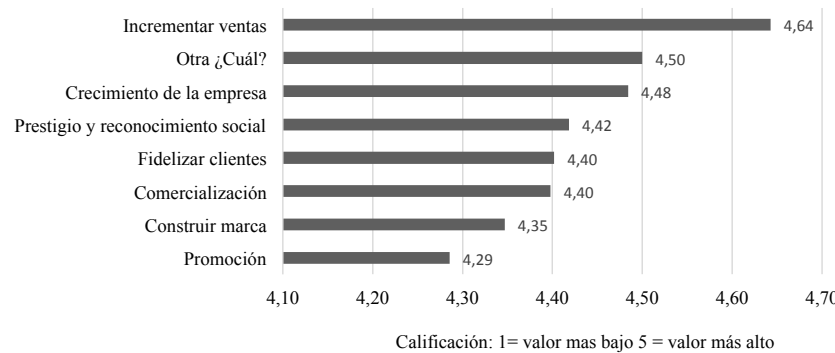

Fuente: elaboración propia. 


\section{CONCLUSIONES}

En Colombia el turismo es un sector emergente con enorme potencial de crecimiento por sus bondades ambientales y socio-económicas, además, porque el país cuenta con una abundante riqueza natural y cultural apta para el turismo sostenible. De manera que las estrategias que permitan convertir dicho patrimonio en productos de clase mundial, aún están en construcción; una de ellas es el modelo de negocio basado en las TIC que ayudará a las MiPymes a mejorar su competitividad permitiendo consolidar los destinos turísticos y darlos a conocer a nivel internacional.

El turismo como sector económico es reciente en el país, contando con apoyo desde los sectores empresarial, público y academia mediante la estrategia "Triada de la competitividad: Universidad-Empresa-Estado", que buscan aunar esfuerzos para consolidar sectores económicos emergentes (Ramírez y García, 2010).

Ahora bien, los hallazgos de la investigación, tanto de fuentes primarias como secundarias permiten evidenciar que existe una gran oportunidad para que las empresas turísticas colombianas apropien las TIC como estrategia de marketing, ya que hay un entorno favorable de cobertura de Internet en el país -para el 2018, el 100\% del territorio nacional, estará conectado a Internet (MinTIC, 2014)- y aunque su presencia en la red es relativamente reciente porque según los resultados del estudio de fuentes primarias: alrededor del $71 \%$ tienen menos de 4 años, la mayoría todavía no tienen estrategias de marketing por Internet suficientemente maduras; sólo el 24\% utilizan aplicaciones especializadas en turismo y alrededor de la mitad de las agencias tienen software especializado, siendo estas empresas las que llevan más de 5 años con presencia en Internet.

Todavía la presencia en Internet de las empresas turísticas colombianas, no es una estrategia de comunicación de marketing consolidada. Sin embargo, el 63\% consideran que ésta es una inversión rentable y el $41 \%$ la tienen como una opción en desarrollo. Los empresarios resaltan que en el uso de TIC han tenido un efecto positivo en aspectos como: llegar más fácilmente a público objetivo, mejorar la competitividad y lograr el mayor conocimiento de la empresa por parte de los clientes. Además consideran que la página web y el correo electrónico son las herramientas que más contribuyen a mejorar las ventas (3.95 y 3.91 respectivamente en una escala de 1-5), mientras que redes sociales y las OTAS (Online Travel Agencies) fueron calificadas con 3.27 y 3.0 respectivamente.

Finalmente, se puede afirmar que aún falta camino por recorrer para que los empresarios de las Mipymes implementen estrategias adecuadas de marketing, debido a lo reciente del sector, al desconocimiento del potencial de las TIC y a la deficiente investigación e innovación. Pero se abre la oportunidad para seguir trabajando en ésta dirección, donde la academia tiene el reto gigante para la formación del capital humano competente a través de la modernización de los planes de estudio de sus programas de pregrado y postgrado y del fortalecimiento de la investigación, el desarrollo y la innovación que permitan encontrar soluciones para que el turismo sea un sector de talla mundial y el país, un destino reconocido. 


\section{REFERENCIAS BIBLIOGRÁFICAS}

AKOUMIANAKIS, D.; VIDAKIS, N.; AKRIVOS, A.; MILOLIDAKIS, G.; KOTSALIS,

D. Y VELLIS, G. (2011): «Building Flexible vacation packages using collaborative assembly toolkits and dynamic packaging: The Case Study of the eKoNES». Journal of Vacation Marketing, vol. $17 \mathrm{n}^{\circ}$ 1, pp. 17-30.

ALCOCER, A. (2011): «El Comercio Electrónico en el Sector Turístico. Aspectos a tener en cuenta», 14 de abril de 2013. Societic - Sociedad y Tecnología. Disponible en: http://goo.gl/FYwWYz

ALVARADO, C. (2013). Negocios rentables por internet, 20 de febrero de 2013. Disponible en: http://goo.gl/vhvkKj

BERNÉ, C.; GARCÍA, M.; GARCÍA, M.E.; MÚGICA, J.M. (2011): «La influencia de las TIC en la estructura del sistema de distribución turístico». Cuadernos de Turismo, $\mathrm{n}^{\mathrm{o}} 28$, pp. 9-22. 22, 15 de marzo de 2015.

BOTERO, J.H.; ZULUAGA, S. y CAMACHO, C. (2010). Estudio de prospectiva para la industria de la hotelería: proyecto de la Asociación Hotelera de Colombia (COTELCO). Bogotá D.C., Fedesarrollo.

BUHALIS, D. (2000): «Marketing the competitive destination of the future», Tourism Management, $\mathrm{n}^{\circ}$ 21, pp. 97-116.

CABERO, V. (2006): «Por una lectura crítica del territorio: repensar el oikoumene», en Sociedad y Medio Ambiente, pp. 181-196. España, Ediciones Universidad.

CASALÓ, L. V., FLAVIÁN, C. y GUINALÍU. M. (2012): «Redes sociales virtuales desarrolladas por organizaciones empresariales: antecedentes de la intención de participación del consumidor». Cuadernos de Economía y Dirección de la Empresa, $\mathrm{n}^{\circ} 15$, pp. 42-51, 16 de agosto de 2016.

CASTRILLÓN, A.J. (2010): El desarrollo turístico del departamento del Cauca (Colombia): una visión desde la academia. Popayán-Colombia, Editorial Universidad del Cauca.

CEREZO, J. (2010): La blogosfera hispana: pioneros de la cultura digital. España, Oman Impresores.

CERVANTES, B., JORGE F. y GÓMEZ, R. (2007): «El ordenamiento territorial como eje de planeación de proyectos de turismo sustentable». Ciencias Sociales Online, vol. IV, $\mathrm{n}^{\circ} 2$, pp. 103-117, 1 de julio de 2015.

CLOPATOFSKY, J. (29 de septiembre de 2013): «Trancones aéreos, reto para los próximos 20 años». El tiempo. Bogotá D.C., 7 de agosto de 2015. Disponible en: http:// goo.gl/tUIZ0h

DANE (2015): Indicadores Básicos de Tendencias y Uso de Tecnologías de la Información y Comunicación en Microestablecimientos. Bogotá-Colombia, Departamento Administrativo Nacional de Estadística (DANE).

DÍAZ, S. (2011): «Congreso Nacional de Agencias de Viajes». Ponencia del Ministro de Comercio, Industria y Turismo. Barranquilla-Colombia, 10 de septiembre de 2014. Disponible en: http://goo.gl/VDyLNY

DÍAZ, O. y LÓPEZ, A.L. (2016): «Comportamiento e interrelación del turismo y el transporte aéreo en Colombia». Estudios y Perspectivas en Turismo, vol. 25, nº 2, pp. 36-56. 
DNP (2010): CONPES 3678. Política de Transformación Productiva: un modelo de desarrollo sectorial para Colombia. Bogotá- Colombia, Departamento Nacional de Planeación.

DNP y MCIT (2011): Plan Sectorial de Turismo 2011 - 2014 Turismo: factor de prosperidad para Colombia, 14 de agosto de 2015. Disponible en: http://goo.gl/BXC2ee

DNP (2005). CONPES 3397: Política Sectorial de Turismo. Bogotá- Colombia, Departamento Nacional de Planeación.

EUROPARC (2007): La Carta Europea del Turismo Sostenible en los Espacios Protegidos. Texto oficial. España, 21 de abril de 2015. Disponible en: http://www.ambientum. com/pdf/carta-europea.pdf

FORMANCHUK, A.L. (2010): Comunicación interna 2.0: un desafío cultural. $1^{a}$. Edición. Buenos Aires, Formanchuk \& Asociados.

GEOFFREY, I. y BRENT J.R. (1999): «Tourism, Competitiveness, and Societal Prosperity». Journal of Business Research, $\mathrm{n}^{\circ}$ 44, pp.137-152.

GÁLVEZ, E.J. (2014): «Tecnologías de Información y Comunicación, e innovación en las MIPYMES de Colombia». Cuadernos de administración, vol. 30, n 51, pp. 71-79.

HERNÁNDEZ, R.; FERNÁNDEZ, C. y BAPTISTA, P. (2010): Metodología de la investigación. $5^{\mathrm{a}}$. Edición. México D.F., McGraw Hill.

JIMÉNEZ, R. (2011): Libro blanco de los viajes sociales: Cómo internet y el protagonismo de los viajeros ha revolucionado el sector turístico, 6 febrero de 2014. Disponible en: http://www.minube.com/libroblanco

LAMSFUS, C.; GRÜN, C.; ALZUA, A. y WERTHNER, H. (2010): «Context-based Matchmaking to enhace Tourist's Experiencies. Monograph: Information Technology in the Tourism Industry». Upgrade, vol. XI, nº 2, pp. 14-21.

LEY 300 (1996): Ley General de Turismo. Bogotá- Colombia. Diario Oficial no 42845 , de julio 30 de 1996.

LÓPEZ, A.L. (2009): «Sistema Integrado de Gestión para empresas turísticas». Estudios Gerenciales, vol. 25, n 112, pp. 131-150.

LÓPEZ, J.M. y LÓPEZ, L.M. (2007): «Diferencias territoriales en la planeación y gestión de los destinos turísticos». Cuadernos de Turismo, $\mathrm{n}^{\circ}$ 19, pp. 71-90.

LÓPEZ, A.L y MARTÍNEZ, O.R. (2015): Las TIC en el sector turístico colombiano. Bogotá - Colombia, Editorial Universidad Autónoma de Colombia.

MELGOSA, F. (2005): «Del desarrollo sostenible al turismo sostenible. La integración del medio ambiente en el sector turístico», en Gestión del medio ambiente, pp. 189-236 Salamanca- España, Universidad de Salamanca.

MEYER, D. (2002): Turismo y Desarrollo Sostenible. Bogotá- Colombia, Universidad Externado de Colombia.

MCIT (2017): «Boletín mensual de turismo. Enero de 2017», 7 de julio de 2017. Disponible en: https://goo.gl/tLc7KF

MCIT (2015a): «Estadísticas de turismo (informes históricos)», 28 de abril de 2015. Disponible en: http://goo.gl/j4JM93

MCIT (2015b): «Informe Turismo Enero 2015», 3 de abril de 2015. Disponible en: http:// goo.gl/DNSgBJ 
MCIT y MINC (2007): Política de turismo cultural: identidad y desarrollo competitivo del patrimonio. Bogotá- Colombia, Ministerio de Comercio, Industria y Turismo y Ministerio de Cultura.

MINTIC E IPSOS NAPOLEÓN FRANCO (2013): «Estudio de consumo digital. Ministerio de TIC-Colombia», 24 de septiembre de 2013. Disponible en: http://goo.gl/1yfC8w

MINTIC (2014): «Plan Vive Digital Colombia 2014-2018», 21 de abril de 2015. Disponible en: http://goo.gl/u9KH5U

MORENO, I. (2007): Manejo integral costero: por una costa más ecológica, productiva y sostenible. Cooperció al Desenvolument i Solidaritat, Vol. 3. Islas Baleares, Planisi S.A.

NIETO, A. (2012): «Las 40 redes sociales más populares». Observatorio de Redes Empresariales de Barrabés América. 25 de febrero de 2013. Disponible en: https://goo.gl/ AWBkRY

OMT (2013): «El turismo internacional mantendrá un crecimiento fuerte en 2013», 4 de febrero de 2013. Disponible en: http://goo.gl/BeFlLi

OMT (2011): «Panorama OMT del turismo internacional». Edición 2011. Organización Mundial del Turismo, 20 de abril de 2013. Disponible en: http://goo.gl/N8Aw8u

OMT (2009): «El turismo al servicio de los Objetivos de Desarrollo del Milenio. Desarrollo Sostenible del Milenio», 3 de enero de 2014. Disponible en: http://goo.gl/yFZwf9

OMT (2001): «Código Ético Mundial para el Turismo». Organización Mundial del Turismo, 20 de febrero de 2015. Disponible en: http://ethics.unwto.org/es/content/ codigo-etico-mundial-para-el-turismo

ORTEGA, F. (2011): La presencia en Internet de las empresas turísticas orientada a la Comercialización Online: herramientas utilizadas y buenas prácticas. Tesis de maestría. Universidad de Sevilla - España, febrero 20 de 2013. Disponible en: https://goo. $\mathrm{gl} / 6 \mathrm{AlZYT}$

PERDOMO, M.A. (2007): «Calidad turística, según la estrategia». Cuadernos de Turismo, $\mathrm{n}^{\mathrm{o}} 10$, pp. 7-27.

PNUMA y OMT (2002): «Declaración de Quebec sobre el ecoturismo», 3 de enero de 2014. Disponible en: http://goo.gl/8qSWhz

PORTER, M. (1991). La ventaja competitiva de las naciones. Barcelona- España, Plaza \& Janes Editores.

QUIRÓS, J. (2003): «El Page Rank de Google», 25 de febrero de 2013. Disponible en: http://goo.gl/UPZl3u

RAMOS, A. y GÓMEZ, A. (2010): Desarrollo del Turismo Interior Comunitario a través de las TIC'S: una oportunidad para la inclusión social y la descentralización. MadridEspaña, Edición AHCIET.

RAMOS, D. (2008): « ¿Quién viaja en avión? Una aproximación a la caracterización de los usuarios europeos del transporte aéreo según su nivel de renta». X coloquio internacional de Geocrítica, 28 de febrero de 2015. Disponible en: http://www.ub.edu/ geocrit/-xcol/102.htm

RAMÍREZ, M.P y GARCÍA, M. (2010): «La Alianza Universidad- Empresa-Estado: una estrategia para promover la innovación». Revista EAN, nº 68, pp. 112-133.

RNT (2013): «Registro de empresas en el Registro Nacional de Turismo», 18 de julio de 2013. Disponible en: http://rnt.rue.com.co/ 
TRIANA, F.; MORALES, J.P.; GUARÍN, A. y SALDARRIAGA, J. (2012). «Mipymes en Colombia. Universidad del Valle- Buga», 3 de octubre de 2014. Disponible en: http://goo.gl/EV5wsJ

TORRES, E. y LASTRA, J. (2008): «Propuesta de una escala para medir la calidad del servicio de los centros de atención secundaria de salud». Revista de Administración Pública-RAP-, vol. 42, $\mathrm{n}^{\circ}$ 4, pp. 719-734.

UNCTAD (2005): «Las TIC y el turismo para el desarrollo». Conferencia de las naciones Unidas sobre el comercio y el desarrollo, 4 de diciembre de 2015. Disponible en: http://unctad.org/es/Docs/c3em25d2_sp.pdf.

VILASECA, J.; TORRENT, J.; LLADÓS, J. y GARAY, L. (2007). «Tecnologías de la información y comunicación, innovación y actividad turística: hacia la empresa en red». Cuadernos de Turismo, ${ }^{\circ}$ 17, pp. 217-240.

YUNIS, E. (2006): «Desafíos de la sostenibilidad en turismo. Implicaciones para la conservación de áreas naturales protegidas». Revista Estudios Turísticos, n 169-170, pp. $77-83$. 